\title{
AVALIAÇÃO DO POSSÍVEL EFEITO BIOESTIMULANTE DO EXTRATO DE ALECRIM NO TEOR DE ÓLEO ESSENCIAL DE
}

\section{Rosmarinus sp.}

\author{
EVALUATION OF THE BIOSTIMULATING EFFECT OF THE EXTRACT OF \\ ROSEMARY ON THE CONTENT OF ESSENTIAL OIL OF Rosmarinus sp.
}

\author{
${ }^{1}$ Lorrane Kelle da Silva Moreira; ${ }^{1}$ Mônyca Laryssa Gomes de Abreu; ${ }^{2,3}$ Maria \\ Juíva Marques de Faria Souza
}

\section{RESUMO}

Introdução: O Rosmarinus sp. (alecrim) possui ação antiinflamatória, antimicrobiana, antitumoral e antioxidante. Os terpenos, flavonóides e ácidos fenólicos (metabólitos secundários) são os responsáveis pelas suas propriedades. Vários fatores interferem no rendimento dos metabólitos secundários, dentre eles está a utilização de bioestimulantes. Objetivo: Avaliar o teor de óleo essencial do Rosmarinus sp. por meio da bioestimulação com o extrato hidro alcoólica de alecrim. Metodologia: Os alecrins em fase adulta foram destinados à produção do extrato hidro alcoólica na concentração de $0,1 \mathrm{~g} / \mathrm{mL}$, e o solvente utilizado foi solução hidro alcoólica a 80\%. Primeiro, o óleo essencial das mudas de alecrim foi extraído pelo método de hidrodestilação acoplado ao aparato de Clevenger, por 2 horas. Posteriormente, cinco mudas de alecrins foram borrifados com extrato de alecrim e cinco com solução hidroalcóolica a 80\%, por 30 dias no período de 08:00 e 13:00h. Em seguida, foram realizadas novas extrações e o teor foi expresso em porcentagem de óleo essencial (\%) em relação à massa fresca. Resultados e Discussão: O teor do óleo essencial de alecrim foi 3,33\%. Após as borrifações, o teor permaneceu o mesmo em todas as plantas. Observou que o extrato apresentou toxicidade sobre as mudas de Rosmarinus sp., pois muitas morreram. Já as mudas que receberam a solução hidro alcoólica a $80 \%$ permaneceram saudáveis. Conclusão: $\mathrm{O}$ extrato hidro alcoólica de alecrim não teve efeito bioestimulante no teor do óleo essencial na espécie Rosmarinus sp., o qual poderá ser uma alternativa no desenvolvimento de herbicidas para o cultivo de lavouras e plantações, devido sua toxicidade.

Palavras-Chave: Alecrim. Bioestimulante. Hidrodestilação.

ABSTRACT: Introduction: The Rosmarinus sp. (rosemary) has an anti-inflammatory action, antimicrobial, antitumor and antioxidant. The terpenes, flavonoids and phenolic acids are responsible for its properties. Several factors interfere in the performance of the secondary metabolites, among these ones is the use of biostimulants. Objective: To

\footnotetext{
${ }^{1}$ Acadêmica do curso de Farmácia, FACER- Faculdade de Ceres, Ceres, Goiás, Brasil.

${ }^{2}$ Docente da FACER- Faculdade de Ceres, Ceres, Goiás, Brasil.

${ }^{3}$ Autor correspondente: Av. Brasil, S/N, Qd. 13 Setor Morada Verde, Ceres-Go. CEP: 763000-000

Fone: (62) 3323-1040. juivamaria@ hotmail.com

Data do recebimento: 10/02/2016

Data da aprovação: 25/03/2016
} 
evaluate the content of the essential oil of Rosmarinus sp. through the biostimulation with the hydroalcoholic extract of rosemary. Methodology: The rosemaries in adult stage were intended for the production of the extract in a concentration of $0.1 \mathrm{~g} / \mathrm{ml}$, and the solvent used was hydroalcoholic solution to $80 \%$. First, the essential oil of rosemary was extracted by the method of hydrodistillation coupled to Clevenger apparatus, for 2 hours. Soon, five sprayed with extract of rosemary and five with hydroalcoholic solution to $80 \%$, for 30 days in the period from 8:00 to 1:00.Then, it was carried out the extractions and the content was expressed as percentage of essential oil (\%) in relation to the fresh mass. Results and Discussion: The yield of the essential oil was $3.33 \%$ of essential oil. After the treatments, there was no increase of the essential oil in none of the plants. It was observed that the extract showed toxicity on the seedlings of Rosmarinus sp., causing the death of the same. On the other hands, the seedlings that received the hydroalcoholic solution to $80 \%$ remained healthy. Conclusion: The hydroalcoholic extract of rosemary had no biostimulating effect on the content of the essential oil in the species Rosmarinus sp., which could be an alternative in the development of herbicides for the cultivation of crops and plantations, due to its toxicity.

Keywords: Rosemary. Biostimulant. Hydrodistillation.

\section{INTRODUÇÃO}

A família Lamiaceae compreende cerca de 300 gêneros e 7500 espécies com distribuição cosmopolita. No Brasil, apresenta cerca de 350 espécies distribuídas em 26 gêneros (SOUZA; LORENZI, 2008). Rosmarinus sp., popularmente conhecido como alecrim, foi uma das primeiras espécies identificadas e classificadas desta família (LORENZI; MATOS, 2008).

O alecrim pode ser utilizado para tratar doenças hepáticas, intestinais, renais, infecções respiratórias, processos inflamatórios e ação antitumoral (BOIX et al., 2010). Também é muito utilizado em fins culinários na forma de condimento para aromatizar os alimentos, e por conter propriedades antimicrobianas e antioxidantes é responsável pela conservação e aumento da vida de prateleira destes (BOIX et al., 2010; ZIBETTI, 2012).

Em diferentes gêneros e espécies de fungos, bactérias e parasitas as ações antimicrobiana e pulgicida do óleo essencial do alecrim têm sido observadas, como por exemplo, estudo de sensibilidade frente ao gênero Cândida (COSTA et al., 2009; CLEFF et al., 2012), ao gênero Salmonella sp. (HENTZ; SANTIN, 2007), às bactérias orais platônicas (SILVA et al., 2008) e a ectoparasitos (BATISTA et al., 2013).

Ressalta-se que a obtenção do óleo essencial de alecrim tem se mostrado uma atividade economicamente importante (SIMÕES et al., 2010). A agroindústria tem investido em pesquisas para que as plantas aumentem a qualidade do óleo essencial, visto que as plantas são valorizadas por suas características aromáticas ou terapêuticas, e que sua matéria-prima pode ser empregada em perfumarias e na indústria química 
(SANGWAN et al., 2001). Ressalta-se, que os óleos essenciais de alecrim são utilizados em larga escala nas indústrias alimentícias, farmacêuticas e de cosméticos (SIMÕES et al., 2010).

As propriedades farmacológicas do alecrim são atribuídas aos metabólitos secundários, os quais têm como função conferir proteção à planta ou permitir que ocorram interações com o meio ambiente (SANTOS, 2010). Segundo Félix-Silva et al. (2012) e Batista et al. (2013) os principais metabólitos secundários presentes no alecrim são os fenóis, como flavonoides, taninos condensados e as saponinas, e os terpenos.

Um dos fatores que contribuem com rendimento dos metabólitos secundários é a utilização de adubos e bioestimulantes de origem vegetal e animal no cultivo de plantas, sendo uma prática utilizada a milhares de anos, o que proporciona o aumento da interação do vegetal com os nutrientes disponíveis (SOUSA et al., 2003; PROBST, 2012).

Os estimulantes de origem vegetal são eficientes em aplicações com doses baixas e favorecem o desempenho dos processos vitais da planta, garantindo que estes sejam satisfatórios (CASILLAS et al.,1986). Os compostos presentes nos estimulantes vegetais são denominados de bioestimulantes, os quais quando aplicados nas plantas reduzem a necessidade de fertilizantes e aumentam a produtividade e a resistência destas ao estresse hídrico e climático (RUSSO; BERLYN, 1990).

Estudo que confirma que os estimulantes de origem vegetal são eficazes foi o trabalho desenvolvido por Dantas e Souza (2014), os quais compararam que o efeito bioestimulante do Ciperus rotundos (tiririca) no desenvolvimento e enraizamento das mudas de Lycopersicon esculentum Mill (tomate) foi superior ao efeito do estimulante químico.

No estudo desenvolvido por Steffen, Antoniolli e Steffen (2010) obtiveram dados satisfatórios no que se refere ao efeito bioestimulante do óleo essencial de eucalipto na germinação e crescimento de mudas de Eucalyptus grandis. Já Bonaldo et. al. (2004) verificou que o extrato de eucalipto no cultivo de pepino foi um agente potencial por conferir resistência ao pepino frente os patógenos. Os autores sugerem que as atividades antimicrobianas presente nas plantas podem contribuir com o desenvolvimento de técnicas de estimulação de origem vegetal, e consequentemente atuar no controle de patógenos responsáveis por distúrbios na planta.

Portanto, de acordo com a busca de informações científicas realizada observou-se que faltam estudos para verificar o rendimento dos metabólitos secundários por meio de 
estimulação de origem vegetal, visto que do ponto de vista farmacêutico são importantes para desenvolvimento de medicamentos.

E por apresentar propriedades antimicrobianas relatadas na literatura científica, o alecrim poderia ser uma alternativa de estimulante de origem vegetal. Logo, diante do exposto, este trabalho teve como objetivo avaliar o teor de óleo essencial do Rosmarinus sp. por meio da possível bioestimulação com o extrato de alecrim.

\section{MATERAIS E MÉTODOS}

\section{Obtenção das mudas de alecrim}

As 17 mudas de alecrim foram adquiridas no viveiro Castro Floricultura na cidade de Itapuranga-Go, e por um período de 30 dias foram cultivadas em sacos plásticos com capacidade de $500 \mathrm{~mL}$, e em seguida foram replantadas em sacos com capacidade para 1 L.

\section{Preparação do extrato hidro alcóolico de alecrim}

Quatro mudas em fase adulta foram destinadas a produção do extrato hidro alcoólico. Primeiramente, coletou as folhas de alecrim, em seguida foram secadas na sombra por 07 dias, e posteriormente rasuradas à mão. Logo, foi preparado um macerado na proporção de $1 \mathrm{~g}$ de droga vegetal seca para $10 \mathrm{~mL}$ de extrato líquido, obtendo uma concentração final de $0,1 \mathrm{~g} / \mathrm{mL}$. O solvente utilizado foi uma solução hidro alcoólica a 80\% (BRASIL, 2010; SIMÕES et al., 2010).

Ressalta-se que a coleta das folhas para as extrações de óleo essencial ocorreu no período da manhã, entre 06:00 e 10:00 horas, visto que o calor do sol poderia levar a perdas quantitativas (MARCHIORI, 2004).

\section{Extração}

Com o objetivo de verificar o possível aumento do teor do óleo essencial da espécie Rosmarinus sp., antes de iniciar os tratamentos foram realizadas extrações por hidrodestilação em aparato do tipo Clevenger por 2 horas (BRASIL, 2010; BARBOSA, et al., 2014), em triplicata de três mudas diferentes com o intuito de analisar se o aumento estaria relacionado com o extrato de alecrim ou com a solução hidro alcoólica à $80 \%$.

Portanto, após coletadas, as folhas frescas de Rosmarinus sp. foram introduzidas no balão de destilação juntamente com água destilada, o qual foi mantido em uma manta 
de aquecimento com temperatura máxima de $100^{\circ} \mathrm{C}$ até atingir fervura. Em seguida, o balão foi acoplado ao aparelho de Clevenger, com o banho de refrigeração à $25^{\circ} \mathrm{C}$. Devido à fotossensibilidade do óleo volátil todo o aparelho foi envolvido com papel alumínio (Figura 1).

Neste método as amostras estavam submersas na água, o vapor d'água arrastou o óleo passando por um condensador, e pela diferença de densidade, óleo e água separaram (SILVEIRA, 2012).

Figura 1: Extração do óleo essencial da espécie Rosmarinus sp. por hidrodestilação em aparato do tipo Clevenger.

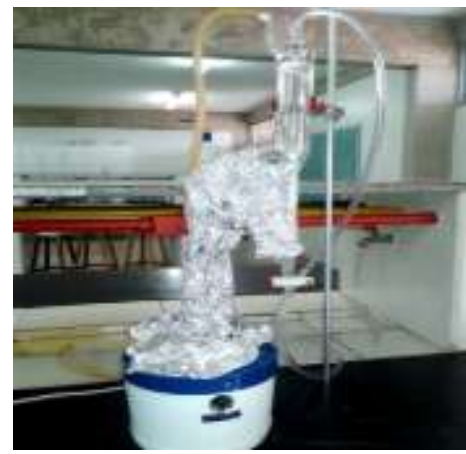

\section{Tratamentos}

Os tratamentos ocorreram no mês de Agosto e Setembro/2015. Nesta etapa as mudas foram dispostas em dois grupos experimentais, em que cinco mudas da espécie Rosmarinus sp. foram borrifadas com extrato hidro alcoólica de alecrim a $0,1 \mathrm{~g} / \mathrm{mL}$ e as cinco mudas restantes foram borrifadas com solução hidro alcoólica a $80 \%$. Este processo ocorreu diariamente às 08:00 e às 13:00 horas, durante 30 dias, até as plantas ficarem embebidas.

Posteriormente, realizou extrações por hidrodestilação em aparato do tipo Clevenger por 2 horas, conforme descrito anteriormente (BRASIL, 2010; BARBOSA, et al., 2014) para verificar o aumento do teor do óleo essencial da espécie em estudo por meio da possível bioestimulação. Todos os óleos essenciais foram coletados em frasco âmbar e armazenado sob refrigeração $\left(20^{\circ} \mathrm{C}\right)$, para estudos posteriores.

Ao final de todas as extrações, o teor foi expresso em porcentagem de óleo essencial (\%) em relação à massa fresca, e os dados obtidos foram submetidos ao Teste $\mathrm{T}$ a $5 \%$ de erro, com $95 \%$ de confiança. 


\section{RESULTADOS E DISCUSSÃO}

Antes de iniciar os tratamentos, com o objetivo de verificar o possível aumento do teor do óleo essencial de Rosmarinus sp. foram realizadas extrações por hidrodestilação em aparato do tipo Clevenger, em triplicata de três mudas diferentes, com o intuito de analisar se o aumento estaria relacionado com o extrato hidro alcoólico de alecrim a $0,1 \mathrm{~g} / \mathrm{mL}$ ou com a solução hidro alcoólica à $80 \%$. Logo, obtiveram a média de 3,33\% de óleo essencial de alecrim. Esse dado foi próximo ao estudo de Barbosa et al. (2014), o qual obtiveram 3,66\% de óleo essencial de alecrim utilizando o mesmo método de extração. Sugere-se que essa pequena variação pode estar relacionada com a sazonalidade das espécies em estudo.

Após os tratamentos, observou-se que não houve aumento significativo do óleo essencial de Rosmarinus sp. em nenhuma das plantas que receberam a bioestimulação com o extrato de alecrim e com as plantas que receberam a solução hidro alcoólica a 80\%, as quais também tiveram $3,33 \%$ de rendimento (Tabela 1 e Tabela 2).

Tabela 1. Resultados das extrações em triplicata por hidrodestilação das mudas da espécie Rosmarinus sp. que receberam tratamento com extrato hidro alcoólica de alecrim a $0,1 \mathrm{~g} / \mathrm{mL}$.

\begin{tabular}{cccc}
\hline Massa fresca $(\mathbf{g})$ & Rendimento de óleo $(\%)$ & Alteração de coloração \\
\hline 1. 3,0050 & $3,33 \%$ & - \\
2. 3,0035 & $3,33 \%$ & - \\
$3 . \quad=$ & $*$ & - \\
\hline
\end{tabular}

Legenda: (=) Sem material suficiente para a extração; (*): Não houve rendimento; (-): Não houve alteração de coloração.

Tabela 2. Resultados das extrações em triplicata por hidrodestilação das mudas da espécie Rosmarinus sp. que receberam tratamento com solução hidro alcoólica a $80 \%$.

\begin{tabular}{ccc}
\hline Massa fresca $(\mathbf{g})$ & Rendimento de óleo $(\mathbf{m L})$ & Alteração de coloração \\
\hline 1. 3,0069 & $3,33 \%$ & + \\
2. 3,0023 & $3,33 \%$ & + \\
3. 3,0050 & $3,33 \%$ & +
\end{tabular}

Legenda: (+): Houve alteração na coloração. 
De acordo com as buscas realizadas, não foi encontrado trabalhos científicos que utilizam bioestimulantes visando o aumento do teor de metabólitos secundários. Em geral, os estudos são voltados para o desenvolvimento, crescimento e proteção das plantas. Isso foi demonstrado no estudo realizado por Itako et al. (2008), no qual o extrato de alecrim diminui em $20 \%$ o número de lesões da pinta preta (Alternaria solani) em folhas de tomateiro. Em contrapartida, o estudo desenvolvido por Gardiano et al. (2011) avaliou o efeito do extrato aquoso de alecrim na proporção de $1 \mathrm{~g}$ de folhas secas para cada 10mL de água destilada sobre a multiplicação do nematóide Rotylenchulus reniformis em plantas de algodoeiro, porém não houve resultado significativo.

Foi observado que ao longo do tratamento algumas mudas de Rosmarinus sp. sofreram alterações (Figura 2, Figura 3, Figura 4 e Figura 5). No grupo de plantas que receberam borrifações com extrato hidro alcoólico de alecrim, três morreram. Conforme observado na Tabela 1 foi impossível de realizar a última extração por falta de material vegetal.

Figura 2: Primeira semana de tratamento das mudas da espécie Rosmarinus sp. com extrato hidro alcoólico de alecrim a $0,1 \mathrm{~g} / \mathrm{mL}$ e solução hidro alcoólica a $80 \%$.

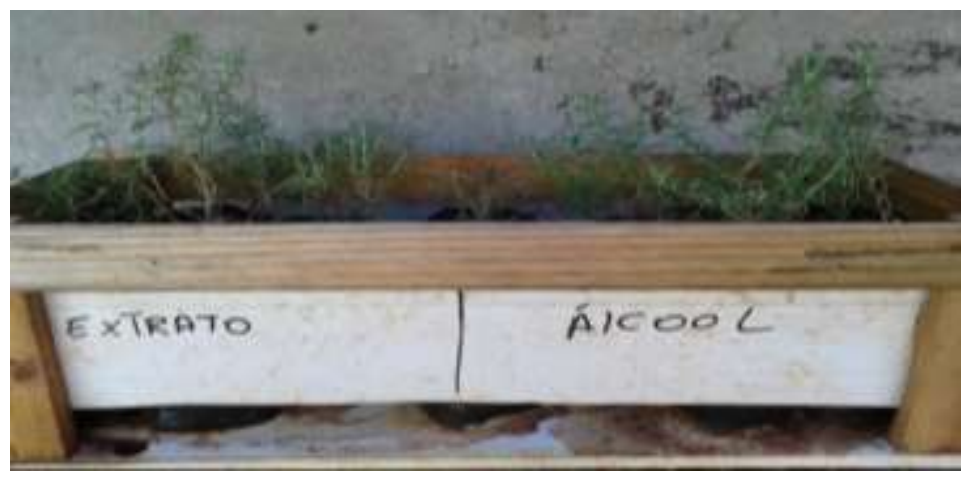

Figura 3: Segunda semana de tratamento das mudas da espécie Rosmarinus sp. com extrato hidro alcoólico de alecrim a $0,1 \mathrm{~g} / \mathrm{mL}$ e solução hidro alcoólica a $80 \%$.

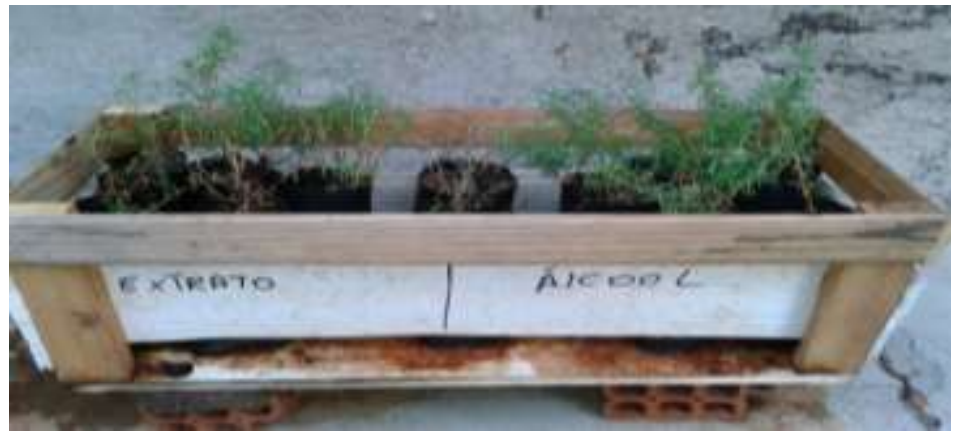


Figura 4: Terceira semana de tratamento das mudas da espécie Rosmarinus sp. com extrato hidro alcoólica de alecrim a $0,1 \mathrm{~g} / \mathrm{mL}$ e solução hidro alcoólica a $80 \%$.

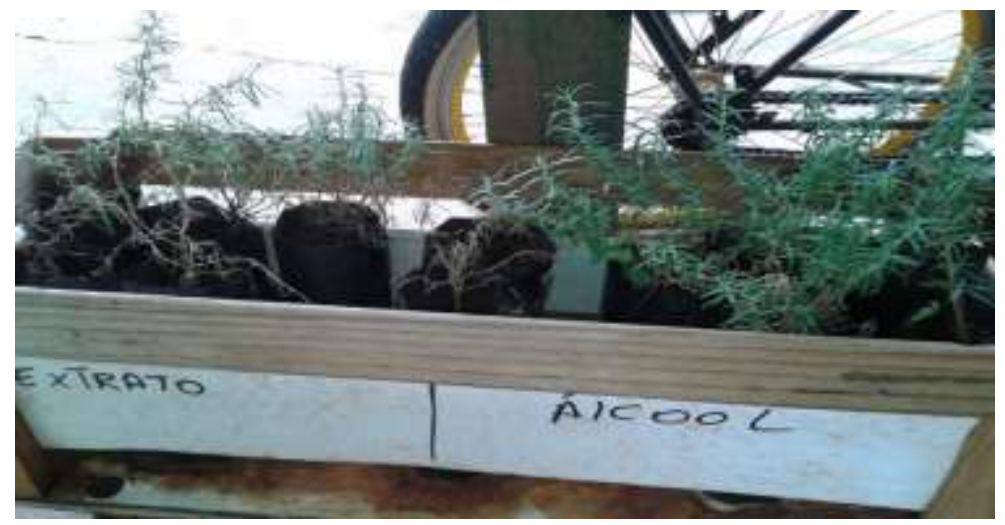

Figura 5: Quarta semana de tratamento das mudas da espécie Rosmarinus sp. com extrato hidro alcoólica de alecrim a $0,1 \mathrm{~g} / \mathrm{mL}$ e solução hidro alcoólica a $80 \%$.

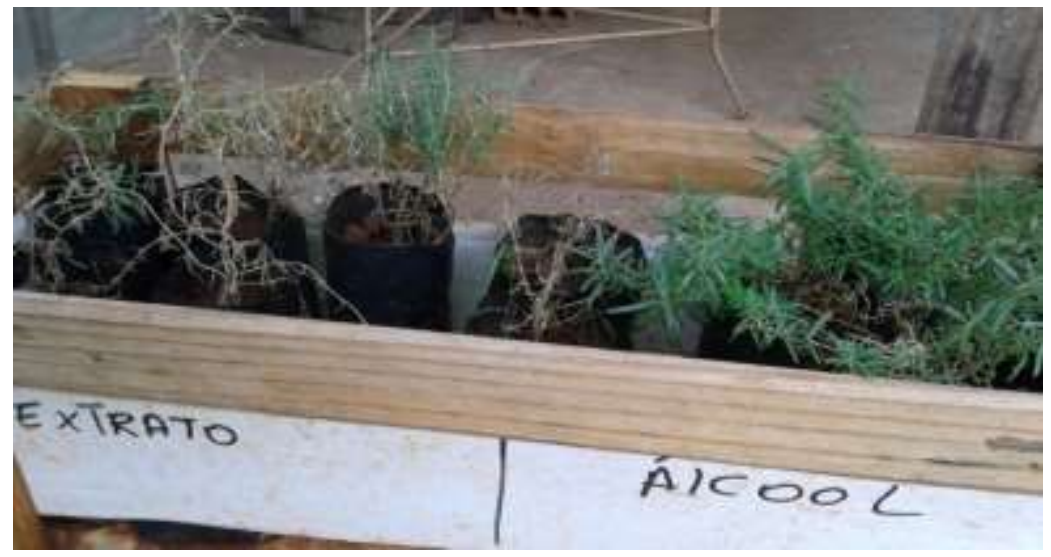

A não ocorrência do possível aumento do teor do óleo essencial de Rosmarinus sp. poderia estar relacionada com a concentração inicial do extrato hidro alcoólica utilizada no experimento, uma vez que, após 30 dias algumas mudas morreram.

Ressalta-se que a morte das mudas de alecrim pôde ser visualizada, a qual iniciouse da raiz para as folhas, talvez foi o local em que se encontrava a maior concentração do extrato hidro alcoólica de alecrim. Portanto, propõe-se para posteriores estudos diminuir a concentração e aumentar o tempo de análise.

Sugere-se que a morte dessas mudas esteja relacionada com certo grau de toxicidade do extrato hidro alcoólica de alecrim, visto que no estudo desenvolvido por Almeida et al. (2009) e por Miolo (1996) observaram certo grau de toxicidade do extrato das folhas de alecrim-pimenta (Lippia sidoides) e do alecrim-do-campo (Vernonia nudiflora), respectivamente. Segundo Almeida et al., (2009), há necessidade de estudos 
na área de toxicidade voltada para plantas, pois trarão importante contribuição para utilização segura e eficaz dos medicamentos provenientes dos mesmos. Portanto, no presente estudo o extrato hidro alcoólica de alecrim também não contribui com o desenvolvimento, crescimento e proteção da espécie Rosmarinus sp.

Já as mudas que receberam a solução hidro alcoólica a $80 \%$ permaneceram saudáveis. Porém, observou alteração na coloração do óleo essencial de alecrim, a qual mudou de amarelo-dourado para incolor. No presente estudo sugere-se que essa alteração esteja relacionada com o estímulo da solução hidro alcoólica a 80\%. Segundo Marchiori (2004) e Santos (2010) há vários fatores externos que podem promover tal alteração, visto que a diferença de solo, condições climáticas, tipo de cultivo, época da colheita e idade da planta, tempo de exposição ao sol e vento, e até mesmo o método de extração dos componentes pode influenciar na concentração e na composição destes óleos.

\section{CONCLUSÃO}

O extrato hidro alcoólica de alecrim a $0,1 \mathrm{~g} / \mathrm{mL}$ foi utilizado como uma possível alternativa de bioestimulantes para aumentar o teor de óleo essencial do Rosmarinus sp. De acordo com os dados apresentados não houve aumento no teor do óleo essencial, e o extrato hidro alcoólica de alecrim apresentou certo grau de toxicidade sobre as mudas de Rosmarinus sp., ocasionando a morte das mesmas. Portanto, estudos posteriores devem ser realizados para verificar o possível aumento do teor do óleo essencial, visto que o óleo essencial de alecrim é muito utilizado na indústria farmacêutica, cosmética e alimentícia. Ou ainda, o alecrim pode ser uma alternativa no desenvolvimento de herbicidas para o cultivo de lavouras e plantações, devido sua toxicidade.

\section{REFERÊNCIAS}

ALMEIDA, A. C. et al. Toxicidade aguda dos extratos hidro alcoólica das folhas de alecrim-pimenta, aroeira e barbatimão e do farelo da casca de pequi administrados por via intraperitoneal. Ciência Rural, v.40, n.1, 2009.

BARBOSA, V. et al. Avaliação da atividade antibacteriana do óleo essencial de Rosmarinus officinalis L. e tintura de própolis frente à bactéria causadora da acne Propionibacterium acnes. Revista Brasileira de Plantas Medicinais, v.16, n.2, p.169173, 2014. 
BATISTA, L. C. S. O. et al. Rosmarinus officinalis (Lamiaceae): atividade in vitro frente a ectoparasitos de importância veterinária. Revista Brasileira de Medicina Veterinária, v.35, Supl.2, p.119-125, 2013.

BOIX, Y. F. et al. Volatile compounds from Rosmarinus officinalis L. and Baccharis dracunculifolia DC. Growing in southeast coast of Brazil. Química Nova, v.33, n.2, 2010.

BONALDO, et. al. Fungitoxicidade, atividade elicitora de fitoalexinas e proteção de pepino contra Colletotrichum lagenarium, pelo extrato aquoso de Eucalyptus citriodora. Fitopatologia Brasileira, v. 29, n.2, 2004.

BRASIL. Agência Nacional de Vigilância Sanitária. Farmacopéia Brasileira, $5^{a}$ ed., v.1, p.524, Brasília, 2010.

CASILLAS, J. C. et al. Analisis cuantitativo de la aplicacion de cuatro bioestimulantes en el cultivo del rabano (Raphanuss ativus L.). Acta Agronómica, v. 36, n.2, p.185·195, 1986.

CLEFF, M.B. et al.Perfil de suscetibilidade de leveduras do gênero Candida isoladas de animais ao óleo essencial de Rosmarinus officinalis L. Revista Brasileira de Plantas Medicinais, v.14, n.1, p.43-49, 2012.

COSTA, A. C. B. P. et al. Atividade antifúngica dos extratos glicólicos de Rosmarinus officinalis Linn. E Syzygium cumini Linn. sobre cepas clínicas de Candida albicans, Candida glabratae Candida tropicalis. Revista de Odontologia da UNESP, v.38, n.2, p.111-116, 2009.

DANTAS, J. P. S.; SOUZA, F. M. L. Avaliação do desenvolvimento e enraizamento de clones de Lycopersicon esculentum Mill com utilização de estimulante natural. Revista Eletrônica da Faculdade de Ciências Sociais e Agrárias de Itapeva, 4a ed., 2014. Disponível em: < http://fait.revista.inf.br/site/c/engenharia-florestal.html >. Acesso em: 28 Mai 2015.

FÉLIX-SILVA, J. et al. Identificação botânica e química de espécies vegetais de uso popular no Rio Grande do Norte, Brasil. Revista Brasileira de Plantas Medicinais, v.14, n.3, p.548-555, 2012.

GARDIANO. C. G. et al. Efeito de extratos aquosos de espécies vegetais sobre a multiplicação de Rotylenchulus reniformis Linford \& Oliveira. Arquivos do Instituto Biológico, São Paulo, v.78, n.4, p.553-556, 2011. 
HENTZ, S. M.; SANTIN, N. C. Avaliação da atividade antimicrobiana do óleo essencial de alecrim (Rosmarinus officinalis L.) contra Salmonella sp. Evidência, v.7, n.2, p.93$100,2007$.

ITAKO, A. T. et al. Atividade antifúngica e proteção do tomateiro por extratos de plantas medicinais. Tropical Plant Pathology, vol.33, n.3, p.241-244, 2008.

LORENZI, H.; MATOS, F. J. A. Plantas medicinais no Brasil: nativas e exóticas. $2^{\mathrm{a}}$ ed. Nova Odessa: Instituto Plantarum, 2008.

MARCHIORI, V. F. Rosmarinus officinalis. 2004. 32 f. Monografia (Curso de Fitomedicina) - Fundação Herbarium. Associação Argentina de Fitomedicina, Argentina, 2004.

MIOLO, J. R. Intoxicação experimental com alecrim Vernonia nudiflora em ovinos ovis aries. Revista da FZVA, v. 2/3, n. 1, p. 24-29. 1996.

PROBST, I. S. Atividade antibacteriana de óleos essenciais e avaliação de potencial sinérgico. 2012. 112 f. Dissertação (Mestrado em Biologia Geral e Aplicada) - Instituto de Biociências de Botucatu, Universidade Estadual Paulista, Botucatu, São Paulo, 2012.

RUSSO, R. O.; BERLYN, G. P. The use of organic biostimulants to help low input sustainable agriculture. Journal of sustainable agriculture, v.1, n.2, p.19-42, 1990.

SANGWAN, N. S. et al. Regulation of essential oil production in plants. Plant Growth Regulation, v.34, n.1, p. 3-21, 2001. Disponível em: <http://link.springer.com/article/10.1023/A\%3A1013386921596\#page-2>. Acesso em: 23 Nov 2015.

SANTOS, R. I. Metabolismo básico e origem dos metabólitos secundários. In: SIMÕES, C. M. O. et al. Farmacognosia: da planta ao medicamento. $6^{\mathrm{a}}$ Ed. Porto Alegre: Editora da UFRGS; Florianópolis: Editora da UFSC, 2010.

SILVA, M. S. A. et al. Atividade antimicrobiana e antiaderente in vitro do extrato de Rosmarinus officinalis Linn. sobre bactérias orais planctônicas. Revista Brasileira de Farmacognosia, v.18, n.2, p.236-240, 2008.

SILVEIRA, C. J. et al. Levantamento e análise de métodos de extração de óleos essenciais. Enciclopédia Biosfera, v.8, n.15; p.2.038, 2012. 
SIMÕES, C. M. O.; SPITZER, V. Óleos voláteis. In: SIMÕES, C. M. O. et al. Farmacognosia: da planta ao medicamento. $6^{\text {a }}$ Ed. Porto Alegre: Editora da UFRGS; Florianópolis: Editora da UFSC, 2010.

SOUSA, A. H. et al. Produção de biomassa na parte aérea da erva cidreira (Melissa ssp.) em função de doses de esterco bovino, húmus de minhoca, composto orgânico e NPK em casa de vegetação. Revista de biologia e ciências da terra, v.3, n.2, 2003.

SOUZA, V. C,; LORENZI, H. Botânica Sistemática: Guia ilustrado para identificação das famílias de Fanerógamas nativas e exóticas no Brasil. $2^{\mathrm{a}}$ ed. São Paulo: Nova Odessa, Instituto Plantarum, 2008.

STEFFEN, R. B.; ANTONIOLLI, Z. I.; STEFFEN, G. P. K. Efeito estimulante do óleo essencial de eucalipto na germinação e crescimento inicial de mudas de Eucalyptus grandis. Pesquisa Florestal Brasileira, v.30, n.63, p.199-206, 2010.

ZIBETTI, A. W. Desenvolvimento de um processo de separação de compostos bioativos de Rosmarinus officinalis. 2012. 151 f. Tese (Doutorado em Engenharia Química) - Departamento de Engenharia Química e Engenharia de Alimentos, Universidade Federal de Santa Catarina, Florianópolis, 2012. 